\title{
Echocardiographic Assessment of Right Atrial Function in Patients With Myocardial Infarction With Reference to Obstructive Lesions of the Coronary Arteries
}

\author{
Hirohiko Shinomiya, MD; Nobuo Fukuda, MD; Naoki Takeichi, MD; \\ Takeshi Soeki, MD; Hisanori Shinohara, MD; Yasuko Yui, MD; \\ Yoshiyuki Tamura, MD; Takashi Oki, MD*
}

\begin{abstract}
We assessed the relationship between right atrial (RA) function and obstructive lesions of the coronary arteries in 29 patients with recent or old myocardial infarction (MI). Patients were divided into 3 groups according to the location of obstructions as follows: obstruction at the proximal right coronary artery (segments 1 and 2) (RCA proximal group, $n=9$ ); obstruction at the distal RCA (segments 3 and 4) (RCA distal group, $n=6$ ); and obstruction at the left anterior descending coronary artery (LCA group, $n=14$ ). The RA volume and the fractional change in the RA area during atrial contraction (RA \% AC) were evaluated by apical 2-dimensional echocardiography. The right ventricular (RV) end-diastolic pressure (RVEDP) was measured in 4 patients in the RCA proximal group and 4 patients in the LCA group. The ejection fraction of the right ventricle (RVEF) was measured by radionuclide angiography or 2-dimensional echocardiography in 7 patients in the RCA proximal group, 5 patients in the RCA distal group, and 7 patients in the LCA group. The RVEF tended to be lower in the RCA proximal group than in the RCA distal and LCA groups. The RA volume was significantly greater in the RCA proximal group than in the LCA group. The RA \%AC was significantly smaller in the RCA proximal group than in the RCA distal and LCA groups. There were no significant differences in the early diastolic RV inflow velocity among groups, but the late diastolic RV inflow velocity was significantly lower in the RCA proximal group than in the RCA distal and LCA groups. There was no significant difference in the RVEDP between the RCA proximal and LCA groups. Thus, RA dysfunction in the RCA proximal group appeared to be due to myocardial damage rather than to afterload mismatch. These findings suggest that RA dysfunction may occur in patients with an inferior MI who have an obstructive lesion of the proximal RCA. (Jpn Circ J 1998; 62: 393-398)
\end{abstract}

Key Words: Myocardial infarction; Right coronary artery; Right atrial ischemia; Right atrial dysfunction

$\mathbf{G}$ oldstein et $\mathrm{al}^{1}$ reported that dysfunction of the right heart was frequent in an animal model of myocardial infarction (MI) related to obstruction of the right coronary artery (RCA) and that right atrial (RA) function was important for maintaining hemodynamic stability under these conditions. Compensatory augmentation of RA contraction enhances right ventricular (RV) filling and performance in animals and patients with RV infarction, whereas loss of this atrial transport function exacerbates hemodynamic compromise! However, RA function in patients with MI in relation to obstructive lesions of the coronary arteries has not been adequately examined. We examined the relationship between obstructive lesions of the coronary arteries and RA function in patients with recent or old MI.

(Received May 14, 1997; revised manuscript received December 5, 1997; accepted December 18, 1997)

Clinical Research Institute and Division of Cardiology, Zentsuï National Hospital, Zentsuji City, Kagawa

*Second Department of Internal Medicine, Tokushima University School of Medicine, Tokushima, Japan

Mailing address: Nobuo Fukuda, MD, Clinical Research Institute, Zentsuji National Hospital, Senyu-cho 2-1-1, Zentsuji City, Kagawa 765, Japan

\begin{abstract}
Methods
Patient Selection

We studied 29 of 43 patients with MI who were admitted to the Division of Cardiology of our hospital between October 1992 and April 1995 and who underwent coronary angiography (24 men and 5 women, aged 52-77 years, mean 68 years). The diagnosis of MI was based on the clinical history, serial electrocardiographic abnormalities with the development of Q waves $>0.04 \mathrm{sec}$, and an increase in the serum creatine kinase level to more than twice the normal value $(280>\mathrm{IU} / \mathrm{L})$ less than $24 \mathrm{~h}$ after admission. Patients with acute MI and patients in whom evaluable echocardiograms were not obtained were excluded. All subjects were in sinus rhythm. The study population included 2 patients with recent MI in whom the interval from onset until study enrollment was $<1$ month and 27 patients with old MI in whom the interval was $>1$ month. The reason for excluding patients with acute MI from the study is that significant RV dysfunction or tricuspid regurgitation frequently complicates during the acute stage and may affect RA function.
\end{abstract}

\section{Patient Classification}

Patients were divided into groups based on the location of the obstructive lesion of the coronary artery responsi- 
Table 1 Clinical Characteristics of 9 Patients in the RCA Proximal Group

\begin{tabular}{|c|c|c|c|c|c|c|c|c|c|c|c|}
\hline Case & $\begin{array}{c}\text { Age } \\
\text { (years) } \\
\text { and sex }\end{array}$ & $\begin{array}{c}\text { Obstructive } \\
\text { lesion }\end{array}$ & Treatment & Result & $\begin{array}{c}\text { Collateral } \\
\text { circulation }\end{array}$ & $\begin{array}{c}R V I F-E / A \\
(\mathrm{~m} / \mathrm{sec})\end{array}$ & $\begin{array}{c}R A \\
\text { volume } \\
(\mathrm{ml})\end{array}$ & $\begin{array}{c}R A \\
\% A C \\
(\%)\end{array}$ & $\begin{array}{l}\text { RVEDP } \\
(m m H g)\end{array}$ & $\begin{array}{c}R V E F \\
(\%)\end{array}$ & $\begin{array}{c}R V \\
\text { infarction }\end{array}$ \\
\hline 1 & $72 M$ & $\# 1$ & C & - & $L A D \Rightarrow \# 3$ & $0.41 / 0.23$ & 98 & 8 & - & 30 & Yes \\
\hline 2 & $71 M$ & $\# 2$ & $C$ & - & $L A D \Rightarrow \# 3$ & $0.27 / 0.20$ & 74 & 4 & 8 & 27 & Yes \\
\hline 3 & $69 M$ & $\# 2$ & $C$ & - & None & $0.28 / 0.22$ & 56 & 16 & 5 & - & No \\
\hline 4 & $72 F$ & \#2 & $P T C A$ & Success & $L C X \Rightarrow \# 3$ & 0.2910 .37 & 36 & 30 & 5.5 & 45 & No \\
\hline 5 & $58 M$ & $\# 1$ & $P T C A$ & Success & $L C X \Rightarrow \# 3$ & $0.33 / 0.36$ & 38 & 25 & - & 51 & No \\
\hline 6 & $80 F$ & $\# 1$ & $P T$ & Failure & $L A D \Rightarrow \# 2$ & $0.29 / 0.21$ & 45 & 17 & - & - & No \\
\hline 7 & $61 M$ & $\# 1$ & $P T$ & Success & $L C X \Rightarrow \# 3$ & 0.2410 .34 & 48 & 26 & 6 & 37 & No \\
\hline 8 & $69 M$ & $\# 2$ & $P T$ & Success & None & 0.2010 .31 & 40 & 26 & - & 35 & No \\
\hline 9 & $86 M$ & $\# 1$ & $P T$ & Failure & None & $0.33 / 0.24$ & 45 & 20 & - & 32 & No \\
\hline
\end{tabular}

$A$, peak flow vlocity at atrial svstole; $C$, conservative therapy; $E$, peak flow velocity at early diastole; $F$, female; $L A D$, left anterior descending artery; $L C X$, left circumflex artery; $M$, male; PT, peripheral thrombolysis; PTCA, percutaneous transluminal coronary angioplasty; $R V E D P$, right ventricular end-diastolic pressure; $R V E F$, right ventricular ejection fraction; $R V I F$, right ventricular inflow; RA \%AC, percent fractional change of right atrial area; \#1, segment 1; \#2, segment 2; \#3, segment 3.
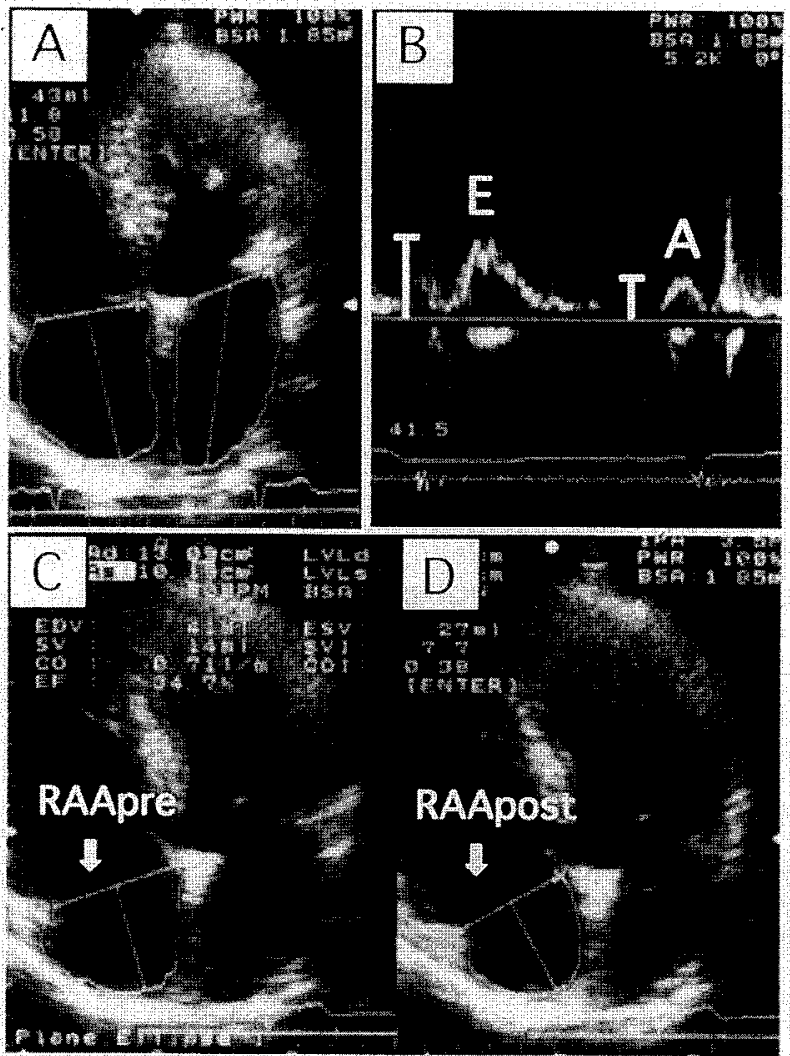

Fig 1. Measurements of atrial volume, right ventricular inflow velocity, and fractional change in the area of the right atrium. (A) Right and left atrial volumes were calculated by the single-plane area-length method from the 2-dimensional echocardiogram of the apical 4-chamber view. LA, left atrium; RA, right atrium. (B) Early (E) and late (A) diastolic peak velocities of the right ventricular inflow were measured from the transtricuspid inflow velocity pattern. (C) RA area immediately before the onset of the ECG P wave (RAA pre) and the area immediately after the ECG R wave (RAA post) were measured from the 2-dimensional echocardiogram of the apical 4-chamber view. The fractional change in the RA area $(\mathrm{RA} \% \mathrm{AC})$ was calculated by the following formula: $\mathrm{RA} \% \mathrm{AC}=(\mathrm{RAA}$ pre-RAA post $) / \mathrm{RAA}$ pre $\times 100$. ECG, electrocardiogram.

ble for the infarction as follows: obstructive lesions in segment 1 or 2 of the RCA according to the American Heart Association (AHA) classification ${ }^{2}$ (RCA proximal group, $\mathrm{n}=9$ ); obstructive lesions in segment 3 or 4 of the RCA (RCA distal group, $n=6$ ); and obstruction at the left anterior descending artery [left coronary artery (LCA) group, $\mathrm{n}=14$ ]. Responsible LCA lesions were detected in segment 6 in 5 patients, segment 7 in 6 patients, and segment 8 in 3 patients. Typical clinical findings of RV infarction during the acute stage were present in 2 of 9 patients with obstructive lesions of the proximal RCA (Table 1). The diagnosis of RV infarction was made from the presence of physical signs indicating right heart failure and low output, the presence of ST-segment elevation in the right precordial leads, and the presence of echocardiographic dilation of the RV cavity.

\section{Acute-Phase Treatment}

In the acute phase, 6 patients underwent percutaneous transluminal coronary angioplasty (PTCA; 2 patients in the RCA proximal group, 1 patient in the RCA distal group, and 3 patients in the LCA group), 13 patients received peripheral thrombolytic therapy (4 patients in the RCA proximal group, 3 patients in the RCA distal group, and 6 patients in the LCA group), and 10 patients received conservative treatment ( 3 patients in the RCA proximal group, 2 patients in the RCA distal group, and 5 patients in the LCA group). The results of acutephase treatment in the RCA proximal group are shown in Table 1.

\section{Two-Dimensional and Doppler Echocardiography}

Two-dimensional and Doppler echocardiograms were recorded using commercially available imaging systems (Aloka SSD-870 and Aloka SSD-2200, Tokyo, Japan) with a $2.5-\mathrm{MHz}$ probe. Left and right atrial echocardiograms were recorded in the apical 4-chamber view with the transducer positioned at the point of the maximal apical impulse and angled toward the right shoulder until all 4 cardiac chambers together with portions of both the mitral and tricuspid valves were visualized. Recordings were made during held expiration. The areas of the left and right atria were calculated by tracing the surface of the endocardium of the left and right atria at the end of ventricular systole. In addition, the volumes of the left and right atria were calculated by the single-plane arealength method (Fig 1A). The fractional change in the RA area (RA \% AC) during atrial contraction was calculated 
using the following formula: (RAA pre-RAA post)/ RAA pre $\times 100(\%)$ (Fig $1 \mathrm{C}$ and D), where RAA pre is the RA area immediately before the $P$ wave on the electrocardiogram and RAA post is the RA area immediately after the $\mathrm{R}$ wave, according to the method advocated by Triposkiadis et al? The RA areas and both atrial volumes were determined by 2 simultaneously working investigators blinded to the conditions of the study. Intraobserver variability in measurements of RA area and atrial volume was $2.9 \pm 2.3 \%$ and $3.1 \pm 2.6 \%$, respectively. Correlation coefficients for measurements obtained by 2 different observers (interobserver variability) were 0.95 for RA area and 0.93 for atrial volume.

Doppler examinations were performed with patients in the left semilateral decubitus position. The ultrasonic beam was transmitted from the lower intercostal space of the left sternal border or near the apex into the RV inflow tract. The sample volume was carefully positioned at the center of the tricuspid annulus on the 2-dimensional echocardiogram. Recordings were performed during held expiration. The peak flow velocities in early diastole $(E)$ and in atrial systole (A) were measured from the baseline to the top of the RV inflow velocity wave (Fig 1B), and the ratio of $\mathrm{A}$ to $\mathrm{E}$ in each cardiac cycle was calculated. In addition, the presence or absence and the severity of tricuspid regurgitation were evaluated by color Doppler echocardiography using the apical 4-chamber or parasternal RV inflow views. The severity of tricuspid regurgitation was determined by the distance of the regurgitant jet from the tricuspid valve orifice, according to the method recommended by Miyatake et al!

Echocardiographic parameters represent the mean of 5 cardiac cycles. The mean intervals from the onset of infarction until echocardiography were 14, 24, and 17 months in the RCA proximal group, the RCA distal group, and the LCA group, respectively.

Serial changes in various echocardiographic and Doppler echocardiographic indices were examined in 4 patients who were followed up immediately after the onset of infarction in the RCA proximal group (mean follow-up 5 months).

\section{Measurements of RV End-Diastolic Pressure and $R V$ Ejection Fraction}

The RV end-diastolic pressure (RVEDP) was measured from the RV pressure curve using a Swan-Ganz catheter in 4 patients in the RCA proximal group and 4 patients in the LCA group. The RV ejection fraction (RVEF) was determined by the multigate method using ${ }^{99} \mathrm{~m} \mathrm{Tc}$ cardiac blood pool scintigraphy in 6 patients in the RCA proximal group, 1 patient in the RCA distal group, and 4 patients in the LCA group, and determined by 2-dimensional echocardiography in 1 patient in the RCA proximal group, 4 patients in the RCA distal group, and 3 patients in the LCA group. On the echocardiogram, the RVEF was calculated by the formula: (RV end-diastolic volume-RV end-systolic volume)/RV end-diastolic volume $\times 100(\%)$, where the RV volume was calculated by the single-plane area-length method at end-diastole and endsystole on the basis of the videotape images of the RV obtained from apical 4-chamber view.

\section{Statistical Analysis}

All values are expressed as means \pm standard deviation (SD). Variables were compared using the non-parametric
Table 2 Clinical, Echocardiographic, Doppler, and Hemodynamic Variables

\begin{tabular}{lccc}
\hline \hline & $\begin{array}{c}\text { RCA prox } \\
(n=9)\end{array}$ & $\begin{array}{c}\text { RCA dist } \\
(n=6)\end{array}$ & $\begin{array}{c}L C A \\
(n=14)\end{array}$ \\
\hline Age (years) & $71 \pm 9$ & $65 \pm 8$ & $68 \pm 12$ \\
Heart rate (beats/m) & $67 \pm 10$ & $65 \pm 12$ & $70 \pm 13$ \\
Atrial volume (ml) & & & \\
LA & $58.4 \pm 24.8$ & $64.5 \pm 27.2$ & $56.2 \pm 18.8$ \\
$R A$ & $53.3 \pm 20.3^{*}$ & $41.2 \pm 6.5$ & $33.6 \pm 8.0$ \\
$R V I F$ & & & \\
$E(\mathrm{~m} / \mathrm{sec})$ & $0.29 \pm 0.06$ & $0.31 \pm 0.09$ & $0.37 \pm 0.09$ \\
$A(\mathrm{~m} / \mathrm{sec})$ & $0.28 \pm 0.07^{* *}$ & $0.36 \pm 0.08$ & $0.37 \pm 0.08$ \\
$E / A$ & $1.06 \pm 0.31$ & $0.90 \pm 0.19$ & $1.02 \pm 0.33$ \\
$R A \% A C(\%)$ & $19.1 \pm 8.7^{* * *}$ & $30.4 \pm 5.6$ & $37.3 \pm 8.0$ \\
$R V E D P(\mathrm{mmHg})$ & $6.1 \pm 1.3$ & - & $6.1 \pm 1.2$ \\
& $(n=4)$ & & $(n=4)$ \\
$R V F F(\%)$ & $36.9 \pm 8.7$ & $45.0 \pm 5.4$ & $45.5 \pm 4.9$ \\
& $(n=7)$ & $(n=5)$ & $(n=7)$ \\
TR $(+)$ & $2 / 9$ & $0 / 6$ & $0 / 14$
\end{tabular}

Values are means $\pm S D .{ }^{*} p<0.01$ vs $L C A,{ }^{* *} p<0.05$ vs $L C A$, $* * * 0<0.05$ vs $R C A$ dist and $L C A$.

$A$, peak flow velocity at atrial systole; dist, distal; $E$, peak flow velocity at early diastole; $L A$, left atrium; $L C A$, left coronary artery; prox, proximal; $R A$, right atrium; $R A \% A C$, percent fractional change of right atrial area; $R C A$, right coronary artery; $R V E D P$, right ventricular enddiastolic pressure; $R V E F$, rigth ventricular ejection fraction; $R V I F$, right ventricular inflow; $T R$, tricuspid regurgitation.

Table 3 Follow-up Data of the 4 Patients in the RCA Proximal Group

\begin{tabular}{|c|c|c|c|c|c|c|}
\hline \multirow[b]{2}{*}{ Case no. } & \multicolumn{2}{|c|}{ RA volume $(\mathrm{ml})$} & \multicolumn{2}{|c|}{$R A \% A C(\%)$} & \multicolumn{2}{|c|}{$R V I F-E / A(\mathrm{~m} / \mathrm{sec})$} \\
\hline & $\begin{array}{l}\text { Acute } \\
\text { phase }\end{array}$ & $\begin{array}{l}\text { Chronic } \\
\text { phase }\end{array}$ & $\begin{array}{l}\text { Acute } \\
\text { phase }\end{array}$ & $\begin{array}{c}\text { Chronic } \\
\text { phase }\end{array}$ & $\begin{array}{l}\text { Acute } \\
\text { phase }\end{array}$ & $\begin{array}{c}\text { Chronic } \\
\text { phase }\end{array}$ \\
\hline 1 & 115 & 98 & 8 & 8 & $0.63 / 0.17$ & 0.4110 .23 \\
\hline 2 & 90 & 74 & 8 & 4 & $0.31 / 0.15$ & $0.27 / 0.20$ \\
\hline 7 & 60 & 48 & 20 & 26 & 0.2910 .25 & 0.2410 .34 \\
\hline 8 & 54 & 40 & 17 & 26 & $0.27 / 0.23$ & 0.2010 .31 \\
\hline
\end{tabular}

Abbreviations are as in Tables 1 and 2.

Mann-Whitney test. Differences were considered statistically significant at $\mathrm{p}<0.05$.

\section{Results}

Comparison of Clinical, Echocardiographic, Doppler, and Hemodynamic Indices Among 3 groups (Table 2)

There were no significant differences in the age and the heart rate among groups. There were no significant differences in the left atrial volume among groups, but the RA volume was significantly greater in the RCA proximal group than in the LCA group and tended to be larger in the RCA proximal group than in the RCA distal group. The early diastolic peak velocity of the RV inflow did not differ significantly among groups. However, the late diastolic peak velocity of the RV inflow was significantly lower in the RCA proximal group than in the LCA group and tended to be lower in the RCA proximal group than in the RCA distal group. The RA \% AC was significantly lower in the RCA proximal group than in the RCA distal group and in the LCA group. Mild tricuspid regurgitation was present in 2 of 9 patients in the RCA proximal group. 



Fig 2. Two-dimensional and pulsed Doppler echocardiographic recordings in the representative patients from the RCA proximal and LCA groups. (A) A 72-year-old man in the RCA proximal group. The right atrial (RA) volume was slightly increased $(74 \mathrm{ml})$. The RA \%AC was markedly decreased $(4 \%)$. The right ventricular inflow velocity in atrial systole (A, $0.20 \mathrm{~m} / \mathrm{sec}$ ) was lower than in early diastole $(\mathrm{E}, 0.27 \mathrm{~m} / \mathrm{sec})$. (B) A 70-year-old man in the LCA group. The RA volume was not increased $(30 \mathrm{ml})$. The $\mathrm{RA} \% \mathrm{AC}$ was increased $(40 \%)$. The right ventricular inflow velocity in atrial systole (A, $0.31 \mathrm{~m} / \mathrm{sec}$ ) was higher than in early diastole (E, $0.22 \mathrm{~m} / \mathrm{sec})$.
Fig 3. Two-dimensional and pulsed Doppler echocardiographic recordings from a 71-year-old man in the RCA proximal group who was followed up for over 4 months after the acute phase of inferior MI. (A) One week after the onset. The right atrial (RA) volume was moderately increased $(115 \mathrm{ml})$. The RA \%AC was markedly decreased (8\%). The right ventricular inflow velocity in atrial systole $(\mathrm{A}, 0.17 \mathrm{~m} / \mathrm{sec})$ is much lower than in early diastole $(\mathrm{E}, 0.63 \mathrm{~m} / \mathrm{sec})$. (B) Four months after the onset. The RA volume was still increased $(98 \mathrm{ml})$. The RA \%AC was still decreased $(8 \%)$. The right ventricular inflow velocity in atrial systole (A, $0.23 \mathrm{~m} / \mathrm{sec}$ ) was still lower than in early diastole $(E, 0.41 \mathrm{~m} / \mathrm{sec})$.
No tricuspid regurgitation was detected in the other 2 groups.

The RVEDP did not differ significantly between the RCA proximal group and the LCA group. The RVEF tended to be lower in the RCA proximal group than in the RCA distal group and the LCA group.

\section{Analysis of the RCA Proximal Group}

Clinical characteristics of 9 patients in the RCA proximal group are shown in Table 1 . There were no significant differences in the RA volume $(54.8 \pm 24.4$ vs $51.5 \pm 17.3 \mathrm{ml})$, the late diastolic peak velocity of the RV inflow $(0.28 \pm 0.07$ vs $0.27 \pm 0.04 \mathrm{~m} / \mathrm{sec})$, or the RA \%AC $(19.2 \pm 7.2$ vs $19.0 \pm 11.6 \%)$ between 5 patients with segment 1 obstruction and 4 patients with segment 2 obstruc- tion. There were no significant differences in the RA volume $(56.5 \pm 24.8$ vs $47.0 \pm 8.2 \mathrm{ml})$, the late diastolic peak velocity of the RV inflow $(0.29 \pm 0.08$ vs $0.26 \pm 0.05$ $\mathrm{m} / \mathrm{sec})$, or the RA \% AC $(18.3 \pm 10.5$ vs $20.7 \pm 5.0 \%)$ between 6 patients with collateral circulation and 3 patients without. The RA volume was significantly greater $(63.6 \pm 22.6$ vs $40.5 \pm 5.3 \mathrm{ml}, \mathrm{p}<0.05)$, the late diastolic peak velocity of the RV inflow was significantly lower $(0.22 \pm 0.02$ vs $0.34 \pm 0.03 \mathrm{~m} / \mathrm{sec}, \mathrm{p}<0.05)$, and the RA $\% \mathrm{AC}$ was significantly smaller $(13.0 \pm 6.7$ vs $26.7 \pm 2.2 \%$, $\mathrm{p}<0.05)$ in 5 patients with conservative treatment or failed peripheral thrombolysis than in 4 patients with successful PTCA or peripheral thrombolysis. The RVEF tended to be lower in 3 patients with conservative treatment or failed peripheral thrombolysis $(29.7 \pm 2.5 \%)$ than 
in 4 patients with successful PTCA or peripheral thrombolysis $(42.2 \pm 7.1 \%)$.

\section{Analysis of the Follow-Up Patients (Table 3)}

Two patients (case 1 and case 2) who were treated conservatively during the acute phase showed no significant improvement in the $\mathrm{RA} \% \mathrm{AC}$ regardless of significant decreases in the RA volume. Another 2 patients (case 7 and case 8) who had successful peripheral thrombolysis during the acute phase showed significant improvements in the $\mathrm{RA} \% \mathrm{AC}$ with concomitant decreases in the RA volume.

\section{Representative Cases}

Fig 2 demonstrates echocardiographic findings in a representative patient from each of the RCA proximal and LCA groups. A 72-year-old man (case 2 in Table 1), who had an obstructive lesion in segment 2 and was treated conservatively, showed an enlarged right atrium (74 ml), a decreased $\mathrm{RA} \% \mathrm{AC}(4 \%)$, and a decreased late diastolic velocity of the RV inflow. A 70-year-old man, who had an obstructive lesion at the left anterior descending artery and was treated conservatively, showed a normal RA volume $(30 \mathrm{ml})$, a normal RA \% AC $(40 \%)$, and a higher late diastolic velocity of the RV inflow.

An apical 2-dimensional echocardiogram in 1 (case 1 in Table 1) of 4 patients in the RCA proximal group, who were followed-up after acute MI, showed significant decreases in the RV and RA sizes 4 months after the onset of MI compared with 1 week after the onset (Fig 3). However, neither the late diastolic peak velocity of the $\mathrm{RV}$ inflow or the RA \% AC were improved at follow-up. Atrial backward velocity of the superior vena cava flow in this patient was decreased 4 months after the onset $(0.06$ $\mathrm{m} / \mathrm{sec})$ as well as 1 week after the onset $(0.05 \mathrm{~m} / \mathrm{sec})$, suggesting that this RV inflow velocity pattern was not a pseudonormalization phenomenon.

\section{Discussion}

Atrial infarction is difficult to diagnose based on clinical findings $5^{-7}$ Autopsy studies have identified atrial infarction in $2.8 \%^{8}$ to $17 \%$ of cases of MI. However, more recent studies ${ }^{10,11}$ have detected atrial involvement, including ischemic lesions, in $65 \%$ of the autopsied cases involving left ventricular posterior wall infarction, and have suggested that atrial infarction involves the right atrium in $90 \%$ of cases ${ }^{10-12}$

Animal experiments have shown that RA contraction is important to the maintenance of RV preload and to the stabilization of hemodynamics during RV infarction!,13 Although case reports have documented the use of transesophageal echocardiography to evaluate RA function during atrial infarction, ${ }^{14,15}$ no studies have systematically examined RA function in patients with MI.

In the present study, the RA volume in the RCA proximal group was larger than in the RCA distal and LCA groups. The rate of reduction in the RA area during atrial contraction and the late diastolic velocity of RV inflow in the RCA proximal group were lower than in the other 2 groups. The increased RA volume in the RCA proximal group may have been due to tricuspid regurgitation and ischemia-related RV and RA dysfunction. However, only mild tricuspid regurgitation was detected by color Doppler echocardiography in 2 of 9 patients in the RCA proximal group. Furthermore, the early diastolic velocity of the $R V$ inflow was decreased in the RCA proximal group, indicating that tricuspid regurgitation was not responsible for the increase in the RA volume.

The decrease in the late diastolic velocity of the RV inflow and the rate of reduction in the RA area during atrial contraction reflected a decrease in the RA contribution to RV filling. There are 2 possible causes of the reduced RA contribution in the RCA proximal group, ie RA systolic dysfunction related to ischemic lesions in the RA and afterload mismatch of the RA as a result of RV dysfunction.

The RVEF measured by cardiac blood pool scintigraphy or 2-dimensional echocardiography was slightly reduced in the RCA proximal group compared with the other 2 groups, suggesting that RV dysfunction was present in patients in the RCA proximal group. In most patients, obstruction at the site proximal to segment 2, where the RV branch diverges, is associated with RV infarction?,10,11,16,17 However, the RVEDP in the RCA proximal group was not significantly higher than in the LCA group, suggesting that RV diastolic dysfunction in the RCA proximal group may not have been marked enough to reduce the RA contribution. Therefore, the reduced RA contribution in the RCA proximal group may have been caused by RA systolic dysfunction related to ischemic lesions in the RA. The results of the present study, in which the RA volume was greater and the RA $\% \mathrm{AC}$ was smaller in 5 patients with conservative treatment and failed peripheral thrombolysis than in 4 patients with successful PTCA and peripheral thrombolysis during the acute phase, support this hypothesis.

RA perfusion is mainly associated with the sinus node artery diverging from segment 1 of the RCA. In addition, atrial branches and intermediate atrial branches diverging from segments 1 and 2 sometimes perfuse the RA 16,17 Chida et $\mathrm{al}^{10}$ and Chida and Ohkawa ${ }^{11}$ reported that atrial ischemic lesions sometimes developed even in patients with RCA obstruction at the site peripheral to the bifurcation of the sinus node artery. In the present study, there were no significant differences in the RA volume, the peak RV inflow velocity, or the rate of reduction in the RA area during atrial systole between RCA proximal subgroups with obstructions in segment 1 or segment 2 .

The present study has some limitations. First, the RVEDP was measured in only 8 patients. However, there was no clinical and echocardiographic evidence indicating right heart failure and $\mathrm{RV}$ dilation in 21 patients without measurement of RVEDP, except for 1 patient (case 1 in Table 1) with complicating RV infarction. Therefore, the RVEDP does not appear to be elevated in these 20 patients. Second, the onset of atrial systole was considered to begin with the $\mathrm{P}$ wave on electrocardiogram. Although atrial systole starts after the $\mathrm{P}$ wave because of the electromechanical delay, the atrial volumes are not expected to change significantly from the onset of the $\mathrm{P}$ wave to the onset of atrial systole 18 The low sampling rate inherent to the echocardiographic examination ( $30 \mathrm{frames} / \mathrm{sec}$ ) is another limitation. Good correlations, however, have been reported between echocardiographic and angiographic measurements as well as between echocardiographic and cine-computed tomographic measurements of atrial volumes 19,20

In conclusion, the present findings suggest that RA dysfunction may occur in patients with MI related to 
obstruction in the proximal RCA involving segments 1 and 2.

\section{References}

1. Goldstein JA, Tweddell JS, Barzilai B, Yagi Y, Jaffe AS, Cox JL: Right atrial ischemia exacerbates hemodynamic compromise associated with experimental right ventricular dysfunction. J Am Coll Cardiol 1991; 18: 1564-1572

2. Austen WG, Edwards JE, Frye RL, Gensini GG, Gott VL, Griffith LSC, et al: A reporting system on patients evaluated for coronary artery disease. Report of the Ad Hoc Committee for Grading of Coronary Artery Disease, Council on Cardiovascular Surgery, American Heart Association. Circulation 1975; 51: 7-40

3. Triposkiadis F, Wooley CF, Boudoulas $\mathrm{H}$ : Left atrial volumes and function in normal subjects. Active and passive emptying. Am J Noninvas Cardiol 1991; 5: 1-6

4. Miyatake K, Okamoto M, Kinoshita N, Ohta M, Kozuka T, Sakakibara $\mathrm{H}$, et al: Evaluation of tricuspid regurgitation by pulsed Doppler and two-dimensional echocardiography. Circulation 1982; 66: $777-784$

5. Hellerstein HK: Atrial infarction with diagnostic electrocardiographic findings. Am Heart $J$ 1948; 36: 422-430

6. Kimura M, Mashiba Y, Kitsuke K, Hagino T, Oonuki H, Katayama $\mathrm{K}$, et al: A pathological study of atrial infarction. A case report. Shinzo 1973; 5: $799-807$

7. Haraoka S, Karai A, Amioka T, Imai M, Inoue S: Atrial infarction. A case report. Ronennbyo 1964; 8: 126-133

8. Horie T, Sekiguchi $M$, Hirosawa K: Atrial infarction and injury. Kokyu to Junkan 1978; 26: $429-436$

9. Cushing EH, Feil HS, Stanton EJ: Infarction of cardiac auricles (atria): clinical, pathological, and experimental studies. Br Heart $J$ 1942; $4: 17-34$
10. Chida K, Ohkawa S, Maeda S, Kuboki K, Imai T, Watanabe C, et al: A clinicopathologic study of atrial infarction complicating left ventricular posterior myocardial infarction. J Cardiol 1992; 22: 1-10

11. Chida K, Ohkawa S: Clinical and pathologic study of atrial infarction. Nippon Rinsho 1994; 52: 588-593

12. Hellerstein HK, Dyke AE: Atrial infarction: a contemporary view. In: Hurst JW, editor. Update IV. The Heart. New York: McGrawHill, 1981: 43-53

13. Kinch JW, Ryan TJ: Right ventricular infarction. $N$ Engl J Med 1994; 28: 1211-1217

14. Hilton TH, Pearson AC, Serota H, Dressler FA, Kern MJ: Right atrial infarction and cardiogenic shock complicating acute myocardial infarction: diagnosis by transesophageal echocardiography. $A m$ Heart $J$ 1990; 120: $427-430$

15. Sasaki T, Matsuzaki M, Anno Y, Tohma Y, Hiroyama N, Uchida T, et al: Diagnosis of right atrial infarction by transesophageal echocardiography. J Cardiogr 1982; 12: 595-604

16. Kawai S, Okada R: Anatomy of coronary arterial system. Nippon Rinsho 1994; 52: 11-26

17. James TN, Burch GE: The atrial coronary arteries in man. Circulation 1958; 17: $90-98$

18. Toma Y, Matsuda Y, Moritani K, Ogawa H, Matsuzaki M, Kusukawa R: Left atrial filling in normal human subjects: relation between left atrial contraction and left atrial early filling. Cardiovasc Res 1987; 21: $255-259$

19. Graham Jr TP, Atwood GF, Faulkner SL, Nelson JH: Right atrial volume measurements from biplane cineangiocardiography. Methodology, normal values, and alterations with pressure or volume overload. Circulation 1974; 49: 709-716

20. Vandenberg BF, Weiss RM, Kinzey J, Stark CA, Stanford W, Burns TL, et al: Comparison of left atrial volume by two-dimensional echocardiography and cine-computed tomography. Am J Cardiol $1995 ; 75: 754-757$ 